\title{
Rate Distortion Analysis and Bit Allocation Scheme for Wavelet Lifting-Based Multiview Image Coding
}

\author{
Pongsak Lasang ${ }^{1}$ and Wuttipong Kumwilaisak ${ }^{2}$ \\ ${ }^{1}$ Media Processing Group, Panasonic Singapore Laboratories (PSL), Block 1022 Tai Seng Avenue 06-3530, Singapore 534415 \\ ${ }^{2}$ Communication and Multimedia Laboratory, Department of Electronics and Telecommunication, Faculty of Engineering, \\ King Mongkut's University of Technology, Thonburi 126 Prachauthis Road, Bangmod, Tungkru, Bangkok 10140, Thailand
}

Correspondence should be addressed to Wuttipong Kumwilaisak, wuttipong.kum@kmutt.ac.th

Received 10 January 2009; Revised 17 May 2009; Accepted 13 August 2009

Recommended by Lisimachos P. Kondi

This paper studies the distortion and the model-based bit allocation scheme of wavelet lifting-based multiview image coding. Redundancies among image views are removed by disparity-compensated wavelet lifting (DCWL). The distortion prediction of the low-pass and high-pass subbands of each image view from the DCWL process is analyzed. The derived distortion is used with different rate distortion models in the bit allocation of multiview images. Rate distortion models including power model, exponential model, and the proposed combining the power and exponential models are studied. The proposed rate distortion model exploits the accuracy of both power and exponential models in a wide range of target bit rates. Then, low-pass and high-pass subbands are compressed by SPIHT (Set Partitioning in Hierarchical Trees) with a bit allocation solution. We verify the derived distortion and the bit allocation with several sets of multiview images. The results show that the bit allocation solution based on the derived distortion and our bit allocation scheme provide closer results to those of the exhaustive search method in both allocated bits and peak-signal-to-noise ratio (PSNR). It also outperforms the uniform bit allocation and uniform bit allocation with normalized energy in the order of $1.7-2$ and $0.3-1.4 \mathrm{~dB}$, respectively.

Copyright (C) 2009 P. Lasang and W. Kumwilaisak. This is an open access article distributed under the Creative Commons Attribution License, which permits unrestricted use, distribution, and reproduction in any medium, provided the original work is properly cited.

\section{Introduction}

In recent years, multiview image coding has become an interesting research area due to its various multimedia applications such as 3-dimensional television, free-viewpoint television, and video surveillance. A set of multiview images is taken by several cameras from different angles. These cameras aim at the same objects to capture the depth of the objects and other useful information. This generates a huge data volume, which makes efficient compression of multiview images necessary.

Most multiview image compression algorithms in literature try to reduce intraview and interview redundancies among image views. In [1], Tong and Gray studied the interview redundancy and the disparity compensated predictive coding for multiview images. Magnor et al. [2] proposed the multiview image coding technique based on the texture map and model-aided prediction. In video coding, the efficient motion compensation schemes using a lifting technique [35] have been proposed to reduce redundancies between video frames, when the wavelet transform is used. It was shown that this guarantees the invertibility at the synthesis side. In addition, using a wavelet compression framework, the scalable property and high-energy compaction can be achieved [3]. The wavelet lifting techniques used for coding multiview images were first proposed in $[6,7]$. The disparity compensation is incorporated into the lifting structure called disparity-compensated lifting to transform the light fields across the views. Haar and 5/3 wavelets are used as the wavelet kernels. The wavelet coefficients in each subband are coded using SPIHT algorithm [8] in a blockwise basis. In [9], Anantrasirichai et al. achieved a spatial scalability of image views via in-band disparity estimation and compensation with the wavelet lifting scheme. The adaptive wavelet lifting framework used for disparity compensation was proposed by Lasang et al. [10]. The mode of disparity compensation 
is selected among Haar, 5/3, or a new proposed wavelet lifting scheme. The criterion in the selection is based on the Minimum Mean Square Error (MMSE) and some selected image features. In their work, the SPIHT codec is also used for coding wavelet coefficients.

To optimally code multiview images with a lifting technique, the bit rate should be allocated to different subbands with the objective to maximize the reconstructed multiview image quality. Without a model, we may need to exhaustively search for the optimal bit allocation solution. This makes the multiview image coding very complex. The first bit allocation algorithm was proposed by Shoham and Gersho [11]. The algorithm provides the optimal solution to the problem for an arbitrary set of quantizers. Since this algorithm needs to compute the rate-distortion (R-D) characteristics for all available quantizers, it has a high computational complexity. The complexity of such algorithm can be significantly reduced, if the R-D characteristics can be accurately approximated. Lin and Ortega [12] used a polynomial-spline function to fit the R-D curve for the optimal bit allocation. In [13], the analytical model is used to approximate the empirical R-D curve. However, the scope of these algorithms is limited to a wide range of bit rate. In [14], Rajpoot investigated the combination of different R-D models to represent the accurate R-D characteristics for a wide range of bit rate. Even though many previous works examine various multiview image coding techniques and the R-D models for encoding image and video contents, there are not many works examining the development of distortion analysis and an R-D model to use in the bit allocation and to code multiview images.

In this paper, we derive the distortion and present the model-based bit allocation scheme for wavelet lifting-based multiview image coding. The derived framework can reduce the complexity in searching for the suitable solution of bit allocation in image subbands. The redundancies among image views are first removed by DCWL. The redundancy removal is performed on the macroblock level with the block size of $16 \times 16$ pixels. The distortion prediction of the low-pass and high-pass subbands of each image view obtained from the DCWL process is analyzed. Together with the derived distortion, a rate distortion model is used in the model-based bit allocation to obtain the bit allocation solution. We study and analyze the accuracy and performance of the model-based bit allocation schemes, when different rate distortion models including exponential model [15], power model [16], and the proposed rate distortion combining both exponential and power models are used. The proposed rate distortion model exploits the accuracy of both models in a wide range of target bit rates. The bit allocation framework allocates bits to all subbands of image views with the goal to minimize distortion of the reconstructed multiview images. Low-pass and high-pass subband components are compressed by SPIHT with the bit allocation solution derived from the model-based bit allocation scheme.

Figure 1 shows the overall framework of the proposed multiview image coding. First the system inputs a set of

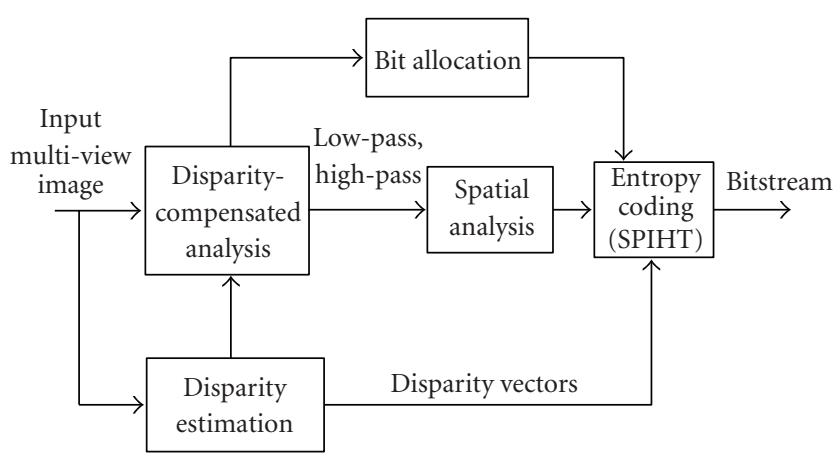

FIgURE 1: The overview framework of the proposed multiview image coding.

multiview images that will be used to encode. Then, blockbased disparity estimation is performed to estimate the disparity vectors. At disparity-compensated (DC) analysis, the estimated disparity vectors are used to compensate the disparity between image views. Then, the wavelet lifting is performed. The wavelet coefficients from DCWL are compressed using SPIHT codec [8]. The bit rate used in SPIHT codec for encoding each subband is computed from the rate distortion model, in which finally the compressed bitstream will be produced.

The remainder of this paper is organized as follows. In Section 2, we present the disparity-compensated wavelet lifting. In Section 3, we analyze and derive the distortion prediction of multiview image, when disparity compensation with the wavelet lifting scheme is used. In Section 4, we describe the model-based bit allocation to different subbands of multiview images based on the derived distortion and different rate-distortion models. The experimental results are shown in Section 5. The conclusion remarks and future works are given in Section 6.

\section{Disparity-Compensated Wavelet Lifting}

The lifting scheme is used to construct the discrete wavelet transform (DWT) as investigated in [17]. In general, highpass $(H)$ and low-pass $(L)$ subband decompositions can be achieved with a sequence of predict $(P)$ and update $(U)$ steps from the lifting structure. There are more than one possible wavelet lifting structures used to code multiview images such as Haar or $5 / 3$ wavelet lifting.

The analysis side of the lifting scheme decomposes multiview images to $H$ and $L$ subbands. Suppose that we have $N$ image views. We divide this group of image views into even views, $X_{2 i}$, and odd views, $X_{2 i+1}$, where $i=0, \ldots,\lfloor N / 2\rfloor$, which are similar and highly correlated in general. In the context of multiview image coding, the disparity estimation and compensation can be effectively integrated into the $P$ and $U$ steps. The synthesis side reconstructs the multiview images from $H$ and $L$ subbands by inverting $U$ and $P$ steps in the analysis side. Figures 2 and 3 show the first level decompositions of the DCWL Haar and 5/3 types, respectively. 


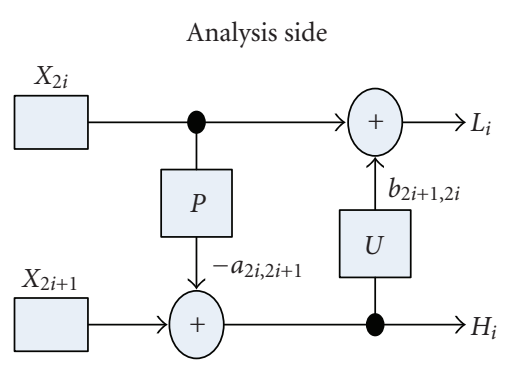

(a)

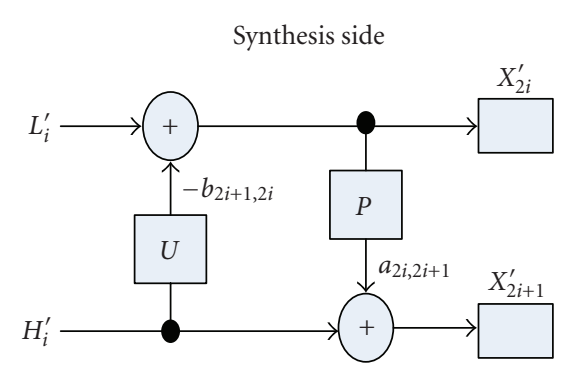

(b)

Figure 2: The first level decomposition of DCWL Haar type.

Analysis side

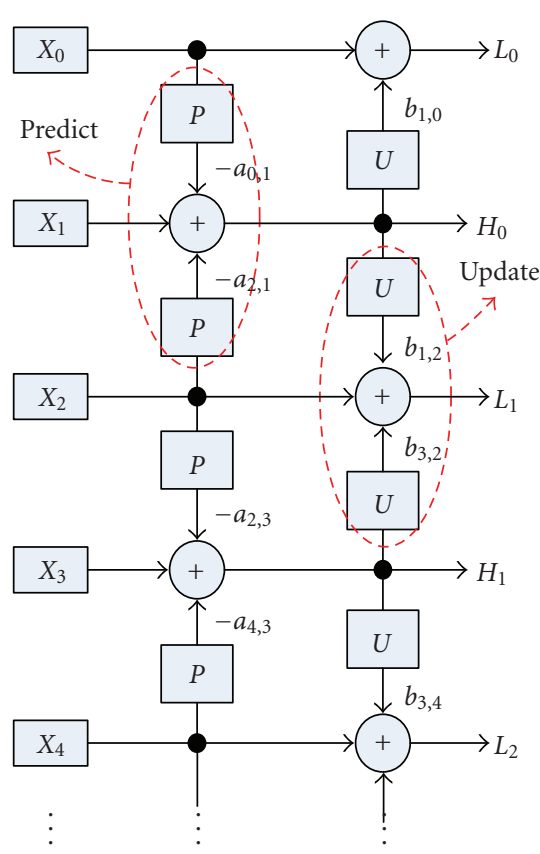

(a)

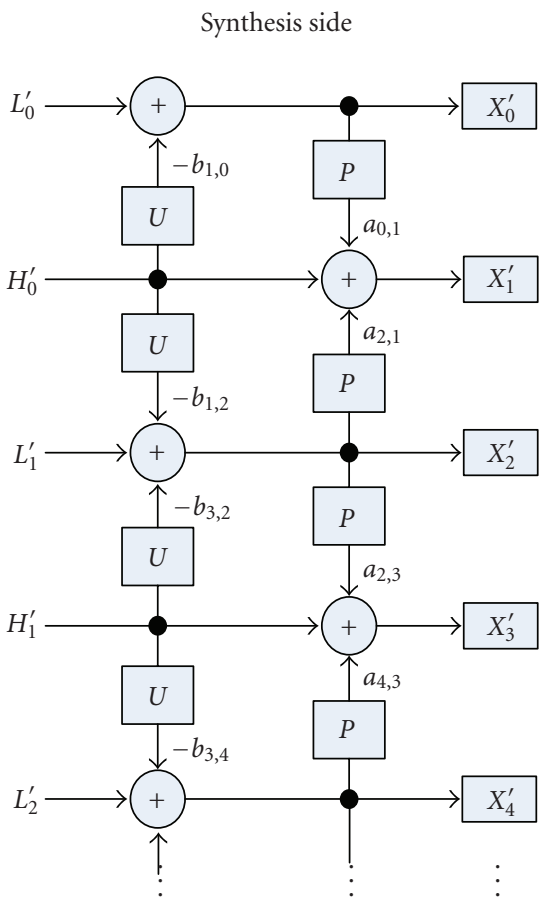

(b)

FIgURE 3: The first level decomposition of disparity compensated 5/3 wavelet lifting.

In the DCWL Haar type, the disparity compensation is performed by using only a single adjacent view as a reference view, whereas $5 / 3$ type uses two adjacent views. Specifically, DCWL Haar type uses image view $i-1$ or image view $i+$ 1 to reduce the redundancy in image view $i$, while DCWL $5 / 3$ type uses both of them. It is possible to use more than two reference image views in DCWL. For example, to predict image view $i$, we may use image views $i-1, i+1, i-3, i+3, i-5$, $i+5, \ldots$. In other words, an even view is predicted from odd views, and an odd view is predicted from even views. In this way, it is guaranteed that all image views can be recovered at the synthesis side of wavelet lifting.

For DCWL Haar type, the $i$ th low-pass $\left(L_{i}\right)$ and high-pass $\left(H_{i}\right)$ components can be written as

$$
\begin{aligned}
H_{i} & =X_{2 i+1}-a_{2 i, 2 i+1} P\left(X_{2 i}, \hat{d}_{2 i+1 \rightarrow 2 i}\right), \\
L_{i} & =X_{2 i}+b_{2 i-1,2 i} U\left(H_{i-1},-\hat{d}_{2 i-1 \rightarrow 2 i}\right) .
\end{aligned}
$$

The $i$ th low-pass $\left(L_{i}\right)$ and high-pass $\left(H_{i}\right)$ components for DCWL 5/3, which uses two reference frames to perform disparity compensation, can be written as

$$
\begin{aligned}
H_{i}= & X_{2 i+1}-a_{2 i, 2 i+1} \times P\left(X_{2 i}, \hat{d}_{2 i+1 \rightarrow 2 i}\right) \\
& -a_{2 i+2,2 i+1} \times P\left(X_{2 i+2}, \hat{d}_{2 i+1 \rightarrow 2 i+2}\right), \\
L_{i}= & X_{2 i}+b_{2 i-1,2 i} \times U\left(H_{i-1},-\hat{d}_{2 i-1 \rightarrow 2 i}\right) \\
& +b_{2 i+1,2 i} \times U\left(H_{i},-\hat{d}_{2 i+1 \rightarrow 2 i}\right),
\end{aligned}
$$

where $\hat{d}_{n \rightarrow m}$ denotes the set of disparity vectors from image view $n$ to image view $m$. $P\left(X_{m}, \hat{d}_{n \rightarrow m}\right)$ is the prediction step, which is the disparity compensation of image $X_{m}$ using $\hat{d}_{n \rightarrow m} . U\left(H_{k},-d_{l \rightarrow j}\right)$ is the update step using $H_{k}$ and inverse disparity vector $-\hat{d}_{l \rightarrow j}$. The scaling factors $a_{m, n}$ and $b_{m, n}$ are 
Table 1: Scaling factors in the $P$ and $U$ steps in different lifting types.

\begin{tabular}{lcc}
\hline Lifting modes & $a_{m, n}$ & $b_{m, n}$ \\
\hline Haar mode & 1 & $\frac{1}{2}$ \\
$5 / 3$ mode & $\frac{1}{2}$ & $\frac{1}{4}$ \\
\hline
\end{tabular}

used in the $P$ and $U$ steps, respectively. All the subscripts, denoting view indices, are restricted between 0 and $N-1$, where $N$ is the number of image views.

We adopt the weighting scheme in [18]. Specifically, $a_{m, n}$ is set to the inverse of the number of reference views and $b_{m, n}$ is set to $a_{m, n} / 2$. Table 1 summarizes the scaling factors in the lifting modes. Figure 4 illustrates the reference image views used in disparity compensation with DCWL Haar and 5/3 types.

At the synthesis side, the inverse $U$ and $P$ steps recover views $X_{2 i}^{\prime}$ and $X_{2 i+1}^{\prime}$ from the subbands. The reconstructed images for DCWL Haar can be written as

$$
\begin{aligned}
X_{2 i}^{\prime} & =L_{i}^{\prime}-b_{2 i-1,2 i} \times U\left(H_{i-1}^{\prime},-\hat{d}_{2 i-1 \rightarrow 2 i}\right), \\
X_{2 i+1}^{\prime} & =H_{i}^{\prime}+a_{2 i, 2 i+1} \times P\left(X_{2 i}^{\prime}, \hat{d}_{2 i+1 \rightarrow 2 i}\right) .
\end{aligned}
$$

The reconstructed multiview images for DCWL 5/3 can be expressed as

$$
\begin{aligned}
X_{2 i}^{\prime}= & L_{i}^{\prime}-b_{2 i-1,2 i} \times U\left(H_{i-1}^{\prime},-\hat{d}_{2 i-1 \rightarrow 2 i}\right) \\
& -b_{2 i+1,2 i} \times U\left(H_{i}^{\prime},-\hat{d}_{2 i+1 \rightarrow 2 i}\right), \\
X_{2 i+1}^{\prime}= & H_{i}^{\prime}+a_{2 i, 2 i+1} \times P\left(X_{2 i}^{\prime}, \hat{d}_{2 i+1 \rightarrow 2 i}\right) \\
& +a_{2 i+2,2 i+1} \times P\left(X_{2 i+2}^{\prime}, \hat{d}_{2 i+1 \rightarrow 2 i+2}\right),
\end{aligned}
$$

where $X_{2 i}^{\prime}$ and $X_{2 i+1}^{\prime}$ are the reconstructed versions of image views $X_{2 i}$ and $X_{2 i+1} . L_{i}^{\prime}$ and $H_{i}^{\prime}$ are the reconstructed versions of low-pass and high-pass subband $i$ of $L_{i}$ and $H_{i}$. Note that at the synthesis side the reconstructed image views may not be equal to those in the analysis side due to the lossy coding by the quantization process or the truncation of wavelet coefficients in each subband.

\section{Distortion Analysis of Wavelet Lifting-Based Multiview Image Coding}

In this section, we analyze the distortion of wavelet liftingbased multiview image coding. In multiview image coding context, to reduce redundancies among image views, the similar pixels from adjacent views are estimated (i.e., disparity prediction in $P$ step). Pixels are classified as "connected pixels," if good matches can be found in the overlapped regions between image views. Otherwise, pixels are classified as "unconnected pixels" as pixels in the nonoverlapped regions in either forward or backward directions. The connected pixels with more than one disparity vectors are known as "multiconnected pixels." These kinds of pixels influence the distortion computation of the reconstructed images. Therefore, their effects are taken into account during the distortion prediction. The example of connected pixels and unconnected pixels between image views 0 and 1 is shown in Figure 5, when parallel views are being captured.

The distortion of reconstructed connected pixels has the influence from multiple reference image views in both forward and reverse disparity prediction, whereas the distortions of reconstructed unconnected pixels have the influences from only reference image views in forward or reverse direction. Let $f$ and $r$ be the ratios of connected pixels in forward and reverse directions of the reference images, where $0 \leq f \leq 1,0 \leq r \leq 1$, and $f+r \leq 1$. Consider the use of the $5 / 3$ wavelet lifting in disparity compensation. First, consider the connected pixel area. Based on Table 1, let $a_{m, n}=a$ and $b_{m, n}=b$ for all $m$ and $n$. The distortion corresponding to the connected pixels of views $X_{2 i}^{\prime}$ and $X_{2 i+1}^{\prime}$ can be expressed as

$$
\begin{aligned}
D_{C, X_{2 i}}= & D_{L_{i}}-b \times\left(D_{H_{i}}+D_{H_{i-1}}\right), \\
D_{C, X_{2 i+1}}= & D_{H_{i}}+a \times\left(D_{X_{2 i}}+D_{X_{2 i+2}}\right) \\
= & D_{H_{i}}+a \times D_{L_{i}}-a \times b \times\left(D_{H_{i}}+D_{H_{i-1}}\right) \\
& +a \times D_{L_{i+1}}-a \times b \times\left(D_{H_{i}}+D_{H_{i+1}}\right) \\
= & (1-2 \times a \times b) \times D_{H_{i}}+a \times D_{L_{i}}-a \times b \times D_{H_{i-1}} \\
& +a \times D_{L_{i+1}}-a \times b \times D_{H_{i+1}},
\end{aligned}
$$

where $D_{C, X_{2 i}}$ and $D_{C, X_{2 i+1}}$ are the distortions in the connected pixel area of image views $X_{2 i}^{\prime}$ and $X_{2 i+1}^{\prime}$, which are caused by forward and backward prediction, respectively. The scaling factor $a$ (predict operator) and the scaling factor $b$ (update operator) are set to $1 / 2$ and $1 / 4$, respectively (for $5 / 3$ wavelet lifting [18]). In this paper, the multiconnected pixels are treated as the regular connected pixels. Therefore, its distortion computation is the same as (5). We also use the same scaling factors $(a=1 / 2, b=1 / 4)$ for the multiconnected pixels. Note that the update operator can be computed based on the number of multiconnected pixels; see [19].

Next, let us consider the distortion in the unconnected pixel area. When only the image views used for forward prediction are used, the distortion of views $X_{2 i}^{\prime}$ and $X_{2 i+1}^{\prime}$ can be written as

$$
\begin{aligned}
D_{U_{f}, X_{2 i}} & =D_{L_{i}}-b_{f} \times D_{H_{i}}, \\
D_{U_{f}, X_{2 i+1}} & =a_{f} \times D_{L_{i}}+\left(1-a_{f} \times b_{f}\right) \times D_{H_{i}},
\end{aligned}
$$

where $D_{U_{f}, X_{2 i}}$ and $D_{U_{f}, X_{2 i+1}}$ are the distortions in the unconnected pixel area of image views $X_{2 i}$ and $X_{2 i+1}$, which are caused by the forward prediction.

When only the image views used for backward prediction are used, the distortion of views $X_{2 i}^{\prime}$ and $X_{2 i+1}^{\prime}$ can be written as

$$
\begin{aligned}
D_{U_{r}, X_{2 i}} & =D_{L_{i}}-b_{r} \times D_{H_{i}}, \\
D_{U_{r}, X_{2 i+1}} & =a_{r} \times D_{L_{i}}+\left(1-a_{r} \times b_{r}\right) \times D_{H_{i}},
\end{aligned}
$$




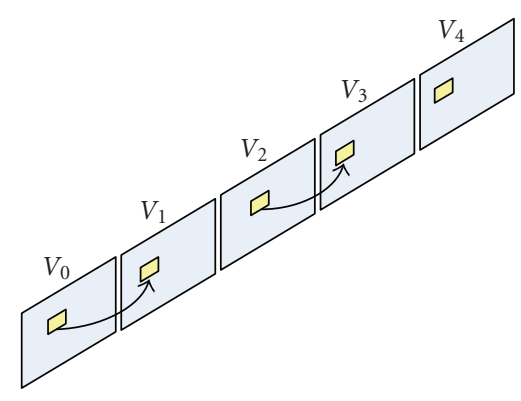

(a) Haar mode

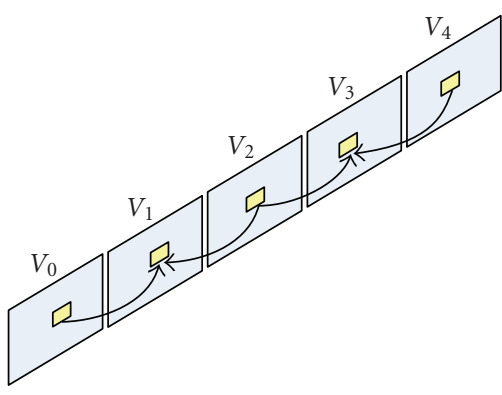

(b) $5 / 3$ mode

FIgURE 4: Illustration of the reference image views in DCWL Haar and 5/3 types.

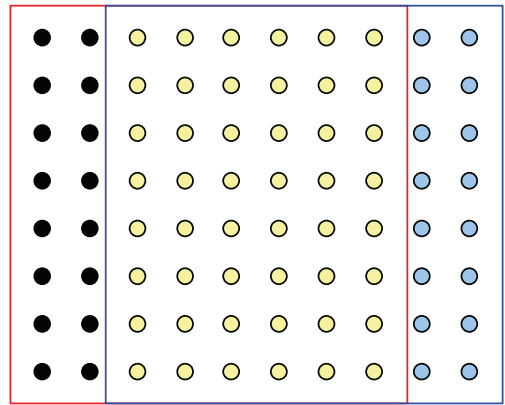

- Unconnected pixels (reverse)

O Connected pixels

Onconnected pixels (forward)

Figure 5: The example shown the unconnected, connected, and occluded pixels, when we consider image view 0 and 1 (reference view).

where $D_{U_{r}, X_{2 i}}$ and $D_{U_{r}, X_{2 i+1}}$ are the distortions in the unconnected pixel area of image views $X_{2 i}$ and $X_{2 i+1}$, which are caused by the backward prediction. The scaling factors $b_{f}$, $a_{f}, b_{r}$ and $a_{r}$ can be computed based on Table 1. More specifically, we set $a_{f}=a_{r}=1$ and $b_{f}=b_{r}=1 / 2$.

We can compute the total distortion of multiview $X_{2 i}^{\prime}$ and $X_{2 i+1}^{\prime}$ from all areas as

$$
\begin{aligned}
D_{X_{2 i}} & =(1-f-r) \times D_{C, X_{2 i}}+f \times D_{U_{f}, X_{2 i}}+r \times D_{U_{r}, X_{2 i}}, \\
D_{X_{2 i+1}} & =(1-f-r) \times D_{C, X_{2 i+1}}+f \times D_{U_{f}, X_{2 i+1}}+r \times D_{U_{r}, X_{2 i+1}} .
\end{aligned}
$$

Assume that every pixel in view $X_{2 i+1}$ can be possibly predicted from two adjacent reference views (view $X_{2 i}$ and $X_{2 i+2}$ ). Based on our observation from several multiview test images, most contents of different image views are close to one another, when cameras are not shifted significantly among image views. Therefore, the disparity compensation can remove redundancy significantly. Based on the fact discussed above, if the distortions of image views are equally distributed, we can write the distortion of $L$ and $H$ subbands as

$$
\begin{aligned}
& D_{H_{k}} \cong D_{H_{k-1}} \cong D_{H_{k+1}}, \\
& D_{L_{k}} \cong D_{L_{k-1}} \cong D_{L_{k+1}} .
\end{aligned}
$$

TABLE 2: The average of $f$ and $r$ ratio of different test images.

\begin{tabular}{lcc}
\hline Test images & Average of $f$ ratio & Average of $r$ ratio \\
\hline Tsukuba & 0.02343750 & 0.01931250 \\
Teddy & 0.02734375 & 0.02539062 \\
Venus & 0.01171875 & 0.02148437 \\
Race1 & 0.0625000 & 0.05468750 \\
\hline
\end{tabular}

The experimental results in Section 5 (see Figure 6) support the above assumption.

From (5), we will obtain

$$
\begin{aligned}
D_{C, X_{2 i}} & =D_{L_{i}}-2 \times b \times D_{H_{i}}, \\
D_{C, X_{2 i+1}} & =2 \times a \times D_{L_{i}}+(1-4 \times a \times b) \times D_{H_{i}} .
\end{aligned}
$$

If we set $a_{f}=a_{r}=a$ and $b_{f}=b_{r}=b$, from (8), the total distortion of multiview $X_{2 i}^{\prime}$ and $X_{2 i+i}^{\prime}$ can be written as

$$
\begin{aligned}
D_{X_{2 i}}= & D_{L_{i}}-(2-f-r) \times b \times D_{H_{i}}, \\
D_{X_{2 i+1}}= & (2-f-r) \times a \times D_{L_{i}} \\
& +(1-(4-3 \times f-3 \times r) \times a \times b) \times D_{H_{i}} .
\end{aligned}
$$

When all blocks can find good matches (i.e., image views are fully overlapped and $f, r=0)$, based on Table $1(a=1 / 2$, $b=1 / 4)(11)$, we can write the total distortions of $X_{2 i}$ and $X_{2 i+1}$ as

$$
\begin{aligned}
D_{X_{2 i}} & =D_{L_{i}}-\frac{1}{2} \times D_{H_{i}}, \\
D_{X_{2 i+1}} & =D_{L_{i}}+\frac{1}{2} \times D_{H_{i}} .
\end{aligned}
$$

For the multiview test images used in this paper, the average ratios of $f$ and $r$ are shown in Table 2 .

Note that the ratios in Table 2 will be used to analyze the distortion and bit allocation of multiview test images in the experimental result.

\section{Rate-Distortion Model and Bit Allocation}

In this section, we study the use of the rate distortion model to perform the bit allocation to the multiview image coding based on the derived distortion in Section 3. 

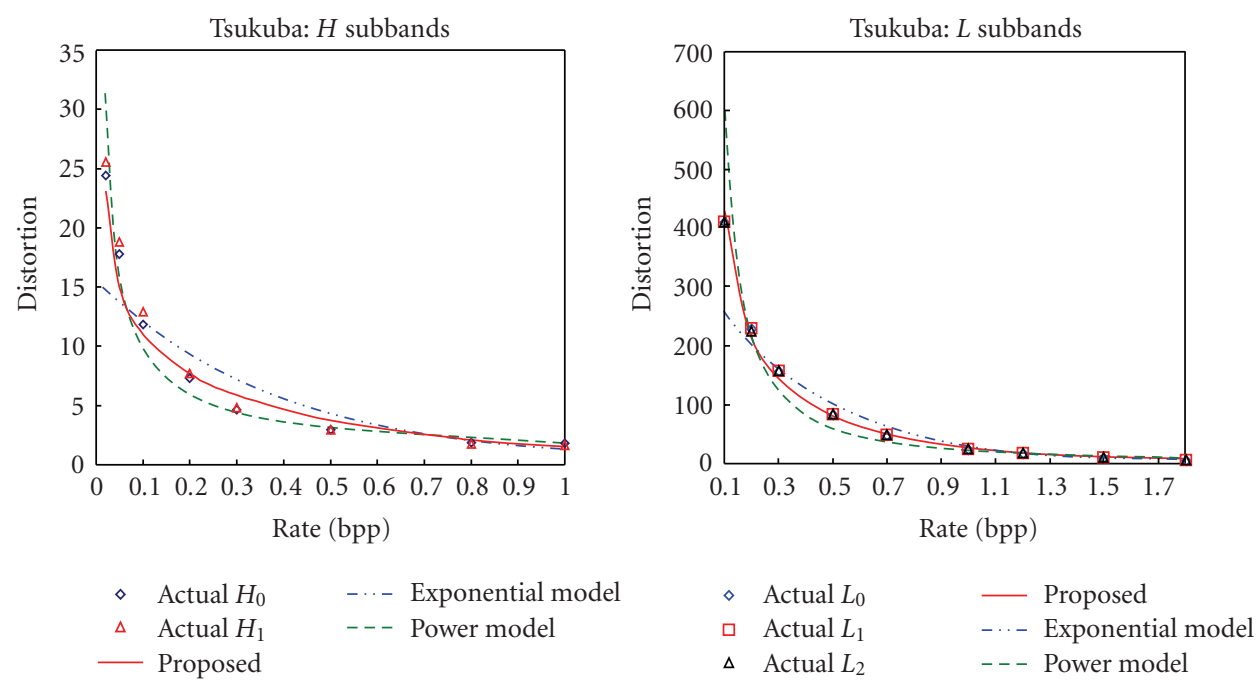

(a)
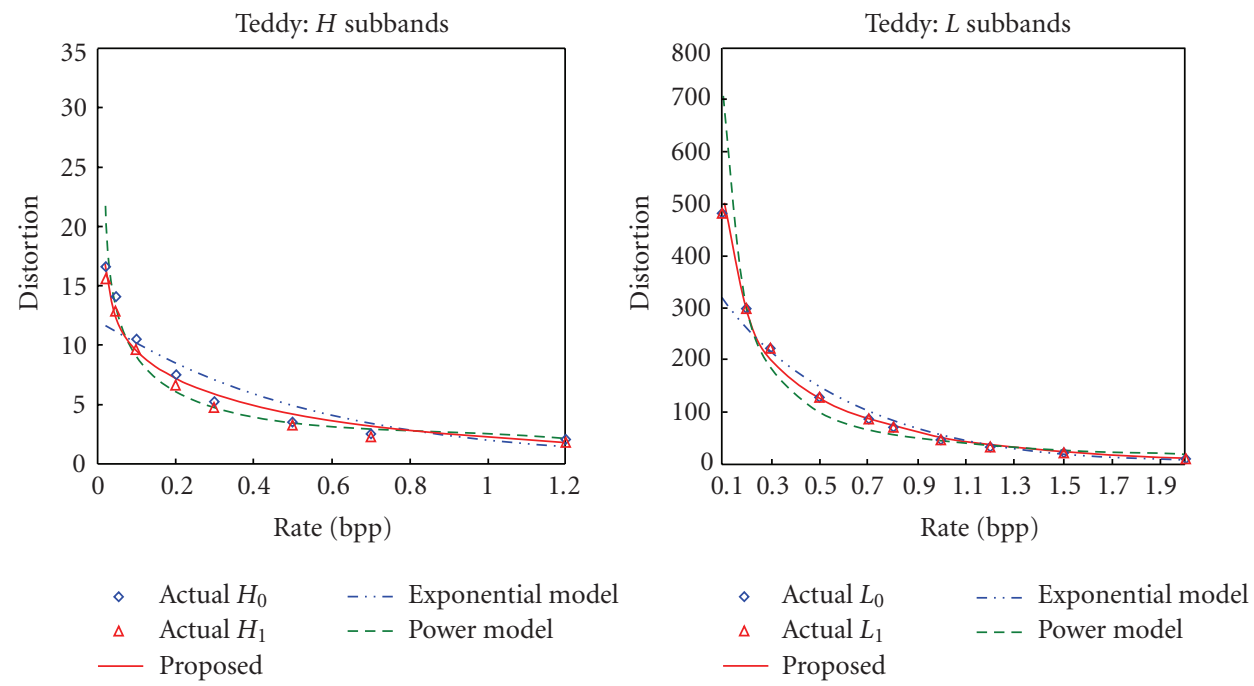

(b)
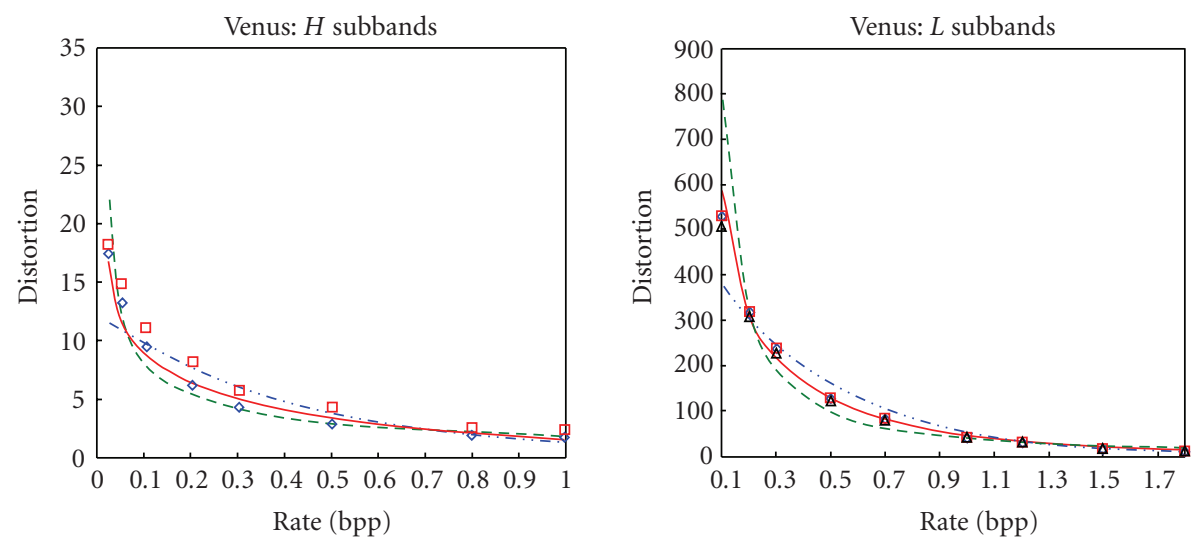

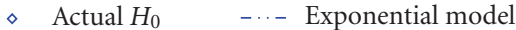

$\square$ Actual $H_{1} \quad---$ Power model

— Proposed
$\diamond$ Actual $L_{0}$
- Proposed
口 Actual $L_{1}$
-..- Exponential model
$\triangle$ Actual $L_{2}$
- - - Power model

(c)

Figure 6: Comparison of the accuracy of the rate distortion models for $H$ subband (left) and $L$ subband (right) of different test sequences (a) Tsukuba, (b) Teddy, and (c) Venus. 
4.1. Rate-Distortion Model. An accurate rate distortion model plays an important role in multimedia compression and transmission due to its efficiency in computation and low complexity. At high bit rate, the exponential model matches well with rate distortion characteristic [16]. If we model the distribution of wavelet coefficients as a Laplacian distribution and define distortion as $D_{e, l}\left(X_{r}, X_{o}\right)=\mid X_{r}-$ $X_{o} \mid$, where $X_{r}$ and $X_{o}$ are the reconstructed image and the original image, respectively, the rate distortion function can be written as [15]

$$
R\left(D_{e, l}\right)=\ln \frac{\sigma}{D_{e, l}} ; \quad 0<D_{e, l}<\sigma,
$$

where $\sigma$ is the standard deviation of wavelet coefficients. $D_{e, l}$ is the distortion from the exponential model, when we model the distribution of wavelet coefficients as a Laplacian source and $R$ is a coding bit rate.

When we model the distribution of wavelet coefficients as a Gaussian distribution and define distortion as

$$
R\left(D_{e, g}\right)=\frac{1}{2} \log \frac{\sigma^{2}}{D_{e, g}} ; \quad 0<D_{e, g}<\sigma^{2},
$$

where $\sigma^{2}$ is the source variance. Laplacian and Gaussian source models are widely used for source modeling because of their mathematical tractability [20]. We can write a general form of the exponential model of both Laplacian and Gaussian sources as [15]

$$
D_{e}(R)=\alpha \times e^{-\beta \times R},
$$

where $D_{e}(R)$ is a general form of the exponential model and $\alpha$ and $\beta$ are the constants depended on the source type.

At a low bit rate region, the power model is highly accurate to represent rate-distortion function [16]. This model can be used for both Gaussian and Laplacian sources. A general form of the power model can be written as

$$
D_{p}(R)=\eta \times R^{-\gamma},
$$

where $\eta$ and $\gamma$ are constants depended on the source type.

However, the exponential model or the power model may not accurately represent the rate-distortion function over a wide range of bit rate. We experimentally compare the accuracy of the exponential and the power models with the actual data using different test images in Section 5 (see Figure 6). We found that both models are not able to fit the actual data in a whole range of bit rate. Therefore, we propose a combined rate-distortion model. It exploits the advantages of both exponential and power models by trying to capture rate distortion function precisely in a whole range of bit rate.

The proposed rate distortion model can be written as

$$
\begin{aligned}
D_{t}(R) & =\omega_{1} \times D_{e}(R)+\omega_{2} \times D_{p}(R) \\
& =\omega_{1} \times \alpha \times e^{-\beta \times R}+\omega_{2} \times \eta \times R^{-\gamma},
\end{aligned}
$$

where $D_{e}(R)$ is the exponential distortion component, $D_{p}(R)$ is the power distortion component, and $\omega_{1}$ and $\omega_{2}$ are the weights of the exponential and the power components, where
$0 \leq \omega_{1} \leq 1,0 \leq \omega_{2} \leq 1$, and $\omega_{1}+\omega_{2}=1 . \alpha, \beta$, $\eta$, and $\gamma$ are the parameters characterizing the proposed distortion model. We compute the parameters $\alpha, \beta$, $\eta$, and $\gamma$ using the least square method, in which we use 7 actual RD points. We observed that the actual R-D points are lined in between the R-D points of the exponential and power models (see Figure 6 in Section 5). Therefore, we empirically set $\omega_{1}=\omega_{2}=0.5$ in this paper as an example for a specific test sequence used in this paper, which give minimum MSE of overall R-D points between the combined model and the actual R-D points. Note that the above choice may not give minimum MSE for other test sequences. Therefore, $\omega_{1}$ and $\omega_{2}$ may be adaptively tuned for low and high bit rate differently depending on the image test sequences.

4.2. Model-Based Subband Bit Allocation. The bit allocation can be formulated as an optimization problem, which aims to minimize the total distortion in a presence of a rate constraint [11]. The total distortion can be expressed as a weighted sum of the distortion of $L$ and $H$ subbands. This is because the distortions from (11) can be written in terms of $D_{L_{k}}$ and $D_{H_{k}}$. The total distortion of all frames can be written as

$$
\sum_{\forall i} D_{X_{2 i}}+\sum_{\forall i} D_{X_{2 i+1}}=\sum_{\forall j} \rho_{L_{j}} \times D_{L_{j}}+\sum_{\forall j} \rho_{H_{j}} \times D_{H_{j}},
$$

where $\rho_{L_{k}}$ and $\rho_{H_{k}}$ are the constants, which are used to weight the distortion between $L$ and $H$ subbands, respectively. With the assumption that the distortion is equally distributed [21], the total distortion can be simplified as

$$
\sum_{\forall i} D_{X_{2 i}}+\sum_{\forall i} D_{X_{2 i+1}}=D_{L} \times \sum_{\forall j} \rho_{L_{j}}+D_{H} \times \sum_{\forall j} \rho_{H_{j}} .
$$

Using (17) as the representation of $D_{L}$ and $D_{H}$, we obtain

$$
\begin{aligned}
& D_{L}=\omega_{1, L} \times \alpha_{L} \times e^{-\beta_{L} \times R_{L}}+\omega_{2, L} \times \eta_{L} \times R_{L}^{-\gamma_{L}}, \\
& D_{H}=\omega_{1, H} \times \alpha_{H} \times e^{-\beta_{H} \times R_{H}}+\omega_{2, H} \times \eta_{H} \times R_{H}^{-\gamma_{H}},
\end{aligned}
$$

where $D_{L}$ and $R_{L}$ are the distortion and the rate of $L$ subband. $D_{H}$ and $R_{H}$ are the distortion and the rate of $H$ subband. Let $R_{\text {total }}$ be the total rate used to code multiview images, let $R_{\mathrm{hd}, \mathrm{DV}}$ be a number of bits used for coding the disparity vectors and header information, and let $R_{\text {texture }}$ be a number of bits used to code the texture information. We know that

$$
R_{\text {texture }}=R_{\text {total }}-R_{\mathrm{hd}, \mathrm{DV}} .
$$

With the definition of distortion and rate described above, the problem in allocating bits to $L$ and $H$ subbands can be formulated as follows.

Problem 1. Given a bit rate constraints $R_{\text {texture }}$ for coding the multiview images, find the optimal bit allocation of $L$ and $H$ subbands such that

$$
\min \left\{D_{L} \times \sum_{\forall k} \rho_{L_{k}}+D_{H} \times \sum_{\forall k} \rho_{H_{k}}\right\},
$$


under the constraint

$$
R_{L} \times \sum_{\forall k} b_{L_{k}}+R_{H} \times \sum_{\forall k} b_{H_{k}} \leq R_{\text {texture }}
$$

where $b_{L_{k}}$ and $b_{H_{k}}$ are the ratios of number of bits in $L_{k}$ and $H_{k}$ subbands.

To facilitate the equations, we define

$$
\begin{aligned}
& f\left(R_{L}, R_{H}\right)=D_{L} \times \sum_{\forall k} \rho_{L_{k}}+D_{H} \times \sum_{\forall k} \rho_{H_{k}}, \\
& g\left(R_{L}, R_{H}\right)=R_{L} \times \sum_{\forall k} b_{L_{k}}+R_{H} \times \sum_{\forall k} b_{H_{k}}-R_{\text {texture }} .
\end{aligned}
$$

We reformulate the problem as

$$
\begin{aligned}
& \min \left\{f\left(R_{L}, R_{H}\right)\right\} \\
& \text { subject to }
\end{aligned}
$$

$$
g\left(R_{L}, R_{H}\right) \leq 0 .
$$

We use a nonlinear programming method [22, 23] to obtain the optimal solution. Therefore (25) can be written as

$$
\min \left\{f\left(R_{L}, R_{H}\right)-\mu \times \sum_{i=1}^{m} \ln s^{(i)}\right\}
$$

subject to

$$
g\left(R_{L}, R_{H}\right)+s=0
$$

where $\mu>0$ is the barrier parameter and the vector of slack variable $s=\left(s^{(1)}, \ldots, s^{(m)}\right)^{T}$ is assumed to be positive.

To compute the optimal bit rate allocation of $L$ and $H$ subbands, we set up a cost function based on the Lagrangian cost function as

$$
\begin{aligned}
& J\left(R_{L}, R_{H}, s, \lambda\right) \\
& \quad=f\left(R_{L}, R_{H}\right)-\left(\mu \times \sum_{i=1}^{m} \ln s^{(i)}\right)+\lambda^{T} \times\left(g\left(R_{L}, R_{H}\right)+s\right),
\end{aligned}
$$

where $\lambda \in \mathfrak{R}^{m}$ are the Lagrange multipliers.

To obtain the optimal solution $\left(R_{L}, R_{H}, s\right)$ of (27), we take partial derivative of (27) respective to $R_{L}$ and $R_{H}$ and we will obtain

$$
\begin{gathered}
\nabla_{R_{L}, R_{H}} J\left(R_{L}, R_{H}, s, \lambda\right)=\nabla f\left(R_{L}, R_{H}\right)+G\left(R_{L}, R_{H}\right) \times \lambda=0, \\
\nabla_{S} J\left(R_{L}, R_{H}, s, \lambda\right)=-\mu \times S^{-1} \times e+\lambda=0,
\end{gathered}
$$

where

$$
G\left(R_{L}, R_{H}\right)=\left(\nabla g^{(1)}\left(R_{L}, R_{H}\right), \ldots, \nabla g^{(m)}\left(R_{L}, R_{H}\right)\right)
$$

is the matrix of constraint gradients, in which superscripts indicating components of a vector, $e=[1, \ldots, 1]^{T}$, and $S=$ $\operatorname{diag}\left(s^{(1)}, \ldots, s^{(m)}\right) . \nabla$ is a derivative operator.

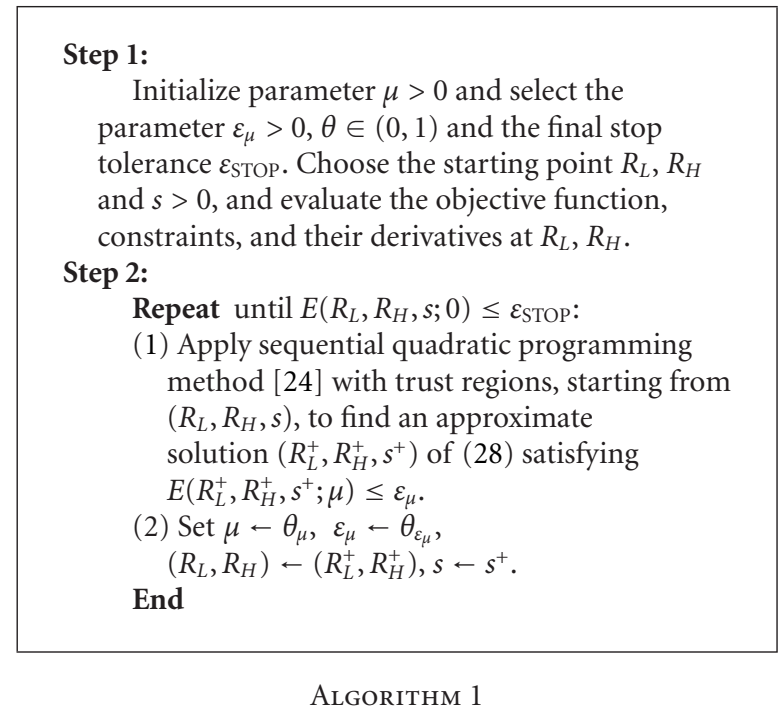

The approximate solution $\left(\hat{R}_{L}, \hat{R}_{H}, \hat{s}\right)$ satisfying $E\left(\widehat{R}_{L}, \hat{R}_{H}\right.$, $\hat{s} ; \mu) \leq \varepsilon_{\mu}$, where $E$ measures the closeness to the optimal solution, is defined by [23]

$$
\begin{aligned}
E\left(R_{L}, R_{H}, s ; \mu\right)=\max \{ & \left\|\nabla f\left(R_{L}, R_{H}\right)+G\left(R_{L}, R_{H}\right)\right\|_{\infty}, \\
& \left.\|S \lambda-\mu e\|_{\infty},\left\|g\left(R_{L}, R_{H}\right)+s\right\|_{\infty}\right\},
\end{aligned}
$$

where \|\|$_{\infty}$ is $L^{\infty}$-norm. The tolerance $\varepsilon_{\mu}$ is decreased from one iteration to the next and must converge to zero. The parameters $\mu$ and $\varepsilon_{\mu}$ will be reduced by a constant factor $\theta \in(0,1)$.

Now, the optimal solution of $R_{L}$ and $R_{H}$ can be derived. The algorithm [23] is described in Algorithm 1.

\section{Experimental Results}

In this section, we present a sequence of experimental results to analyze distortion and bit allocation of multiview images. We use several test multiview images [25] including Tsukuba, Teddy, Venus, and Race1 [26]. Each set of multiview images is composed of 5 image views. The disparity compensation is done in the macroblock level with the size of $16 \times$ 16 pixels. The residue error after the disparity compensation is encoded by SPIHT codec [8]. We utilize the 5/3 wavelet lifting for disparity compensation to demonstrate the developed distortion model and the bit rate allocation.

5.1. Model Accuracy. First, we verify the accuracy of the proposed rate distortion model. We assume that wavelet coefficients obtained from the disparity wavelet lifting have a Laplacian distribution [27]. In our experiments, the actual distortion of reconstructed images is computed for the specific bit rates. Then, we compute the distortion of each specific bit rate from different models (using (15), (16), and (17)) with the same set of bit rate. Figure 6 show the comparison of the accuracy of the proposed rate-distortion 
TABLE 3: The average of the mean square error (MSE) between the actual distortion and the computed distortion of different rate distortion models.

\begin{tabular}{lcccc}
\hline \multirow{2}{*}{ Images } & Subbands & & Average MSE (Mean Square Error) & \\
& & Exponential model & Power model & Proposed model \\
\hline \multirow{2}{*}{ Tsukuba } & $L$ & 2725.079 & 4009.917 & $\mathbf{7 9 . 7 4 7}$ \\
& $H$ & 16.963 & 6.844 & $\mathbf{2 . 3 9 3}$ \\
\hline \multirow{2}{*}{ Teddy } & $L$ & 2702.424 & 5378.732 & $\mathbf{1 6 1 . 2 0 5}$ \\
& $H$ & 4.404 & 4.517 & $\mathbf{0 . 7 1 5 3}$ \\
\hline \multirow{2}{*}{ Venus } & $L$ & 2758.407 & 8469.384 & $\mathbf{4 3 5 . 0 3}$ \\
& $H$ & 7.2376 & 4.4242 & $\mathbf{1 . 8 2 9 5}$ \\
\hline
\end{tabular}

TABLE 4: Comparison of subband bit allocation at target bit rate $0.95 \mathrm{bpp}$.

\begin{tabular}{lcccccc}
\hline Test images & & Uniform allocation & Exponential model & Power model & Proposed & Exhaustive search \\
\hline \multirow{4}{*}{ Tsukuba } & $R_{L_{k}}(\mathrm{bpp})$ & 0.95 & 1.462503 & 1.436343 & 1.377565 & 1.35 \\
& $R_{H_{k}}(\mathrm{bpp})$ & 0.95 & 0.181246 & 0.220485 & 0.308653 & 0.350 \\
& PSNR $(\mathrm{dB})$ & 36.907 & 38.6491 & 38.8251 & $\mathbf{3 9 . 0 4 0}$ & 39.1678 \\
\hline \multirow{3}{*}{ Teddy } & $R_{L_{k}}(\mathrm{bpp})$ & 0.95 & 1.515464 & 1.316667 & 1.414911 & 1.3967 \\
& $R_{H_{k}}(\mathrm{bpp})$ & 0.95 & 0.101804 & 0.4 & 0.252633 & 0.280 \\
& PSNR $(\mathrm{dB})$ & 34.383 & 36.21824 & 36.20671 & $\mathbf{3 6 . 4 3 2 6 7}$ & 36.4482 \\
\hline \multirow{4}{*}{ Venus } & $R_{L_{k}}(\mathrm{bpp})$ & 0.95 & 1.5072 & 1.30173 & 1.6333 & 1.392667 \\
& $R_{H_{k}}(\mathrm{bpp})$ & 0.95 & 0.1142 & 0.4224 & 0.33 & 0.286 \\
& PSNR $(\mathrm{dB})$ & 34.7772 & 36.75062 & 36.59987 & $\mathbf{3 6 . 8 2 0 3 6}$ & 36.8684 \\
\hline \multirow{3}{*}{ Race1 } & $R_{L_{k}}(\mathrm{bpp})$ & 0.95 & 1.460413 & 1.183333 & 1.388267 & 1.360667 \\
& $R_{H_{k}}(\mathrm{bpp})$ & 0.95 & 0.18438 & 0.6 & 0.2926 & 0.334 \\
& PSNR $(\mathrm{dB})$ & 41.4842 & 41.8316 & 42.1802 & $\mathbf{4 2 . 3 7 5 1}$ & 42.4426 \\
\hline
\end{tabular}

model, exponential model, and power model with the actual rate distortion curves of $H$ subband and $L$ subband, when Tsukuba, Teddy, and Venus are used as test images. We can see that the proposed model outperforms the exponential and power models in fitting the rate-distortion curve. Notice that the actual rate-distortion curves of $H_{0}$ and $H_{1}$ as well as the actual distortion curves of $L_{0}$ and $L_{1}$ are close to each other, which verifies the assumption of equally distributed of distortion in Section 3, when the cameras capturing different image views are not shifted significantly from one another. Table 3 shows the average of the mean square error (MSE) between the actual distortion and the computed distortion of different models (range from $0.05 \mathrm{bpp} \sim 1.2 \mathrm{bpp}$ for $H$ subband and $0.1 \mathrm{bpp} \sim 2.0 \mathrm{bpp}$ for $L$ subband). The proposed model gives the minimum MSE comparing to the exponential and power models.

5.2. Bit Allocation Performance. Next, we examine the use of the proposed algorithm in a rate allocation problem. The solution of this rate allocation problem will be used to encode the $H$ and $L$ subbands of multiview images using SPIHT codec. Table 4 shows the comparison on the rate allocation performance comparing the proposed rate distortion model, the exponential model, the power model, the uniform rate allocation, and the exhaustive search rate allocation. The exhaustive search is considered as the best solution. For the exhaustive search, we start with 0.002 bit per pixel and the increment step size is 0.002 bit per pixel. The target bit rate is set to be 0.95 bit per pixel (bpp). As we can see from Table 4, rate allocation using our proposed rate distortion model gives a very close result to the exhaustive search in various test images. Moreover, it outperforms the uniform rate allocation and also uniform rate allocation based on the normalized energy (i.e., proportionally allocate bits to subbands based on their energies) of $L$ and $H$ subbands in terms of average PSNR in the range of $1 \sim 2 \mathrm{~dB}$ and $0.2 \sim$ $0.6 \mathrm{~dB}$ comparing with the exponential and power models. Figures 7 and 8 show the average Peak-Signal-to-Noise Ratio (PSNR) of the reconstructed multiview images of Tsukuba and Teddy images over a wide range of target bit per pixel. The results in the log-log domain of the average PSNR [28] of the reconstructed images are also shown in Figures 9 and 10. We also computed the average gains using the method in [28]. As the results, the proposed method gives $1.7 \sim 2 \mathrm{~dB}$ average gains over the uniform bit allocation, $0.3 \sim 1.4 \mathrm{~dB}$ for the uniform bit allocation with normalized energy, and $0.2 \sim 0.3 \mathrm{~dB}$ average gains over the power and exponential models, respectively. An example of the reconstructed signal ( $H$ and $L$ subbands) of Tsukuba image is shown in Figure 11.

We conclude from the results that the proposed ratedistortion model provides much closer average PSNR results to those using the exhaustive search than the exponential and power models. It also gives significant improvement over the uniform bit allocation almost $2 \mathrm{~dB}$. 


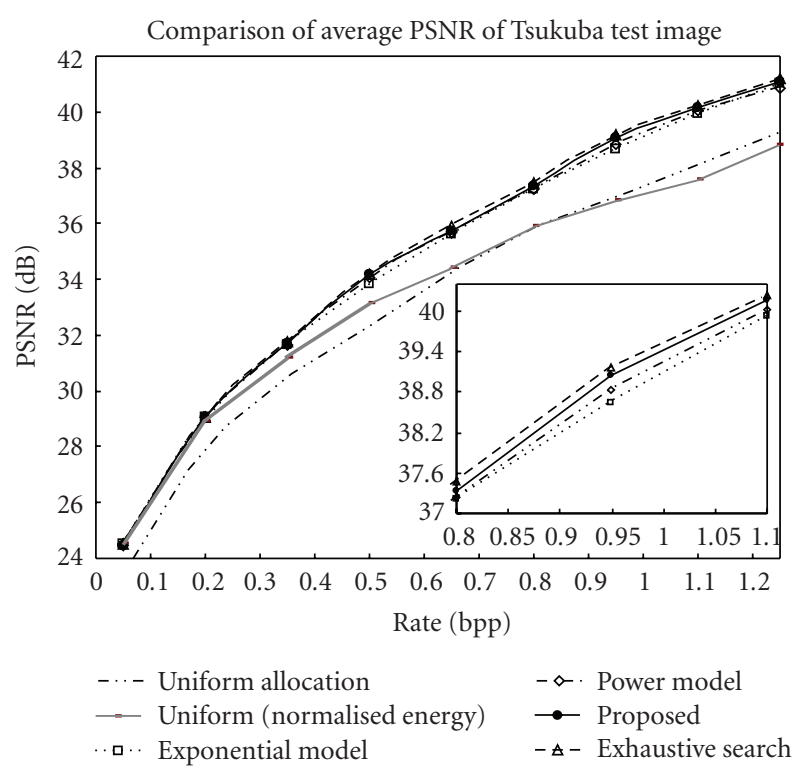

FIGURE 7: PSNR comparison of Tsukuba test image when using different bit allocation methods.

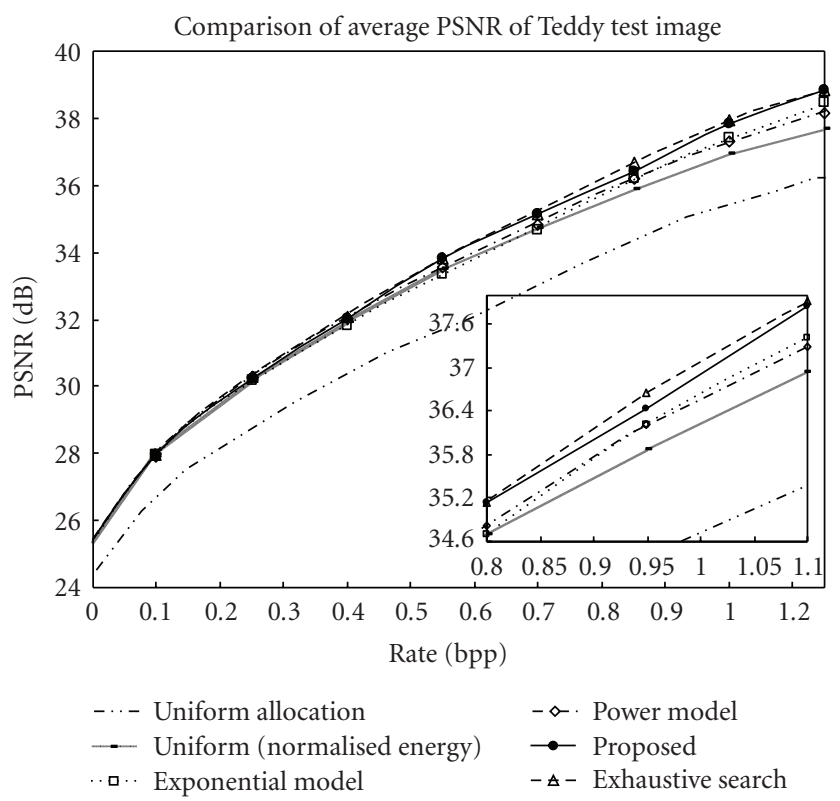

Figure 8: PSNR comparison of Teddy test image when using different bit allocation methods.

5.3. Complexity. We measure the complexity of 5 different bit allocation methods using the processing time. The program was run on the PC with Intel $1.86 \mathrm{GHz}$ CPU and $512 \mathrm{MB}$ of RAM. For each method, we measure the processing time in each submodule. The processing time from different methods is shown in Table 5. The target bit rate is $0.95 \mathrm{bpp}$. Note that the processing time of the common modules, such as disparity estimation and compensation, is not included in the table since all methods are same. Although, from Table 5, the model-based methods require additional processing time for computing 7 actual R-D points and

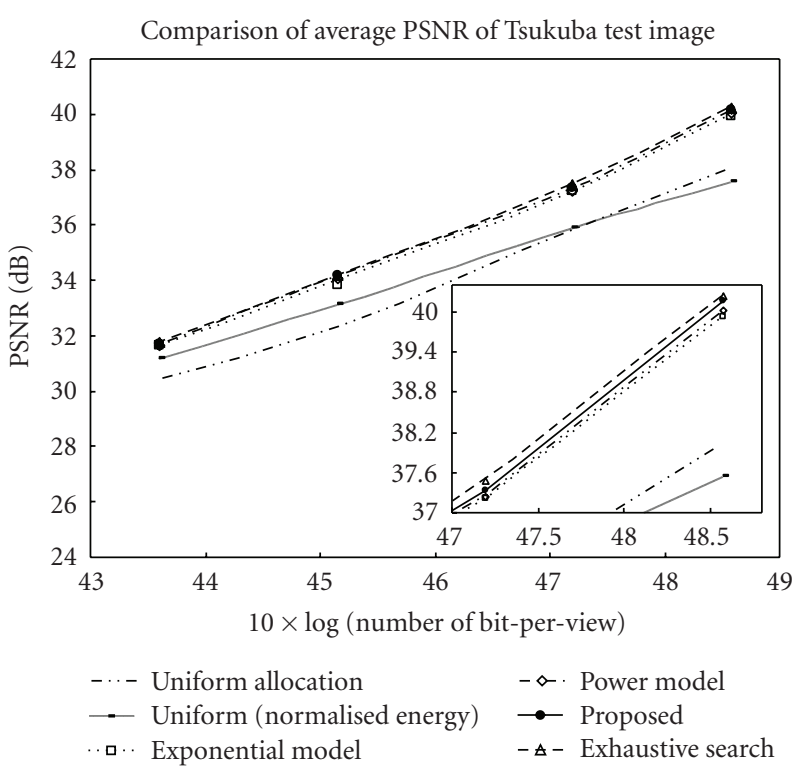

FIGURE 9: PSNR comparison of Tsukuba test image as in Figure 7 using method in [28].

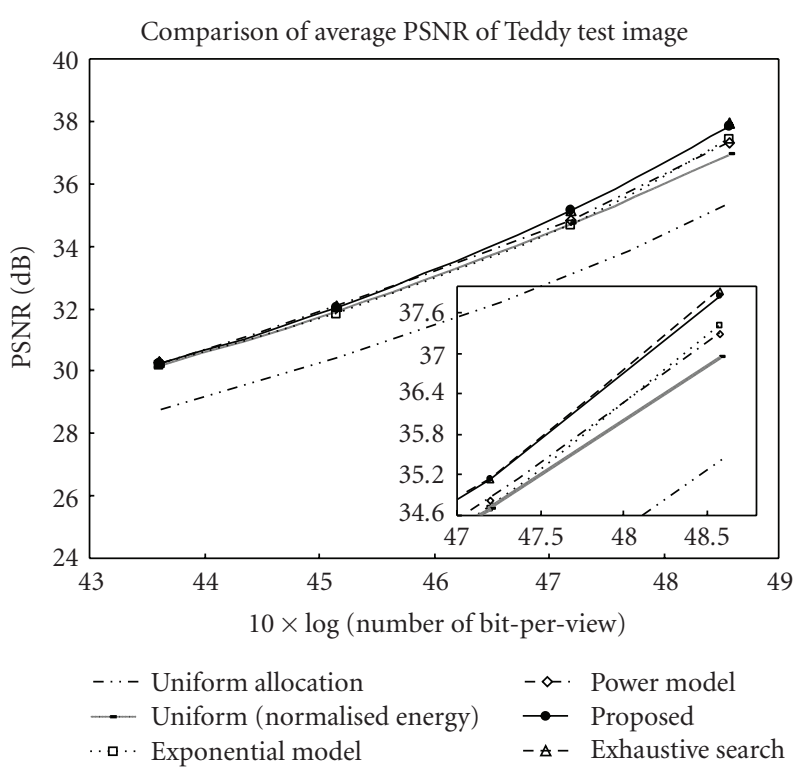

FIGURE 10: PSNR comparison of Teddy test image as in Figure 8 using method in [28].

model parameters, SPIHT encoding/decoding process and synthesis are performed only once. Comparing the proposed model with other two models, the total processing time is almost the same even though the proposed model requires extra time for computing model parameters but it is just a fraction of second.

On the other hands, the exhaustive search method takes up much more processing time. In this paper, we use 4750 points for each given bit rate and search for the allocated bit that gives the best PSNR. This means that the exhaustive search method requires 4750 times of SPIHT 


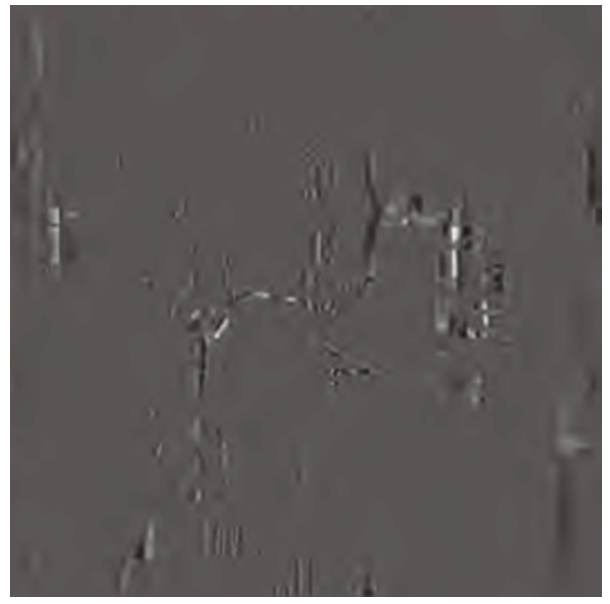

(a)

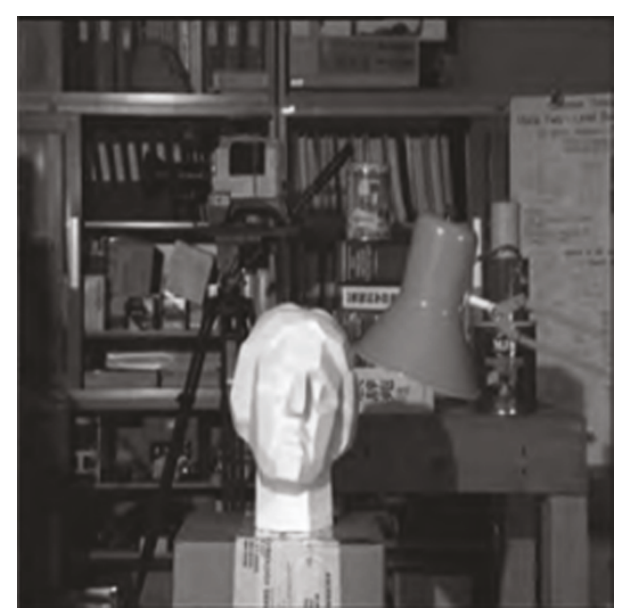

(b)

FIgURE 11: The reconstructed signal of $H$ (a) and $L$ (b) subbands. $H$ and $L$ subbands are encoded with bit rate 0.14 bpp and 0.74 bpp, respectively.

TABle 5: The processing time of different bit allocation methods. The target bit rate is $0.95 \mathrm{bpp}$.

\begin{tabular}{|c|c|c|c|c|c|}
\hline \multirow{2}{*}{ Bit allocation methods } & \multicolumn{5}{|c|}{ Processing time (seconds) } \\
\hline & $\begin{array}{l}\text { Obtaining } 7 \text { actual R-D } \\
\text { points }\end{array}$ & $\begin{array}{l}\text { Computing model } \\
\text { parameters }\end{array}$ & $\begin{array}{l}\text { Obtaining number of bit } \\
\text { to be allocated }\end{array}$ & SPIHT + Synthesis & Total time \\
\hline Uniform & - & - & - & 48.687 & 48.678 \\
\hline Power model & 101.094 & 0.240 & 0.797 & 50.266 & 152.397 \\
\hline Exponential model & 101.094 & 0.261 & 0.750 & 57.391 & 159.496 \\
\hline Proposed & 101.094 & 0.501 & 0.750 & 53.313 & 155.658 \\
\hline Exhaustive search & - & - & 0.112 & 68504.500 & 68504.612 \\
\hline
\end{tabular}

encoding/decoding and synthesis to be processed. From Table 5, the exhaustive search requires processing time 430 times more than the model-based approach to perform bit rate allocation to multiview images.

In addition to the processing time, we also analyze the growth rate of complexity in terms of Big $\mathrm{O}$ notation. We divide the algorithm into two parts: solving optimization problem and SPIHT encoding. The power and exponential models use the same optimization algorithm to allocate bits for $L$ and $H$ subbands and the complexity of the first part of both models is $O(n \log (n / \varepsilon))$, where $n$ is the iteration step and $\varepsilon$ is the tolerance. Thus, the complexity of the combined model is $O(2 n \log (n / \varepsilon))$. For the second part, the complexity of the SPIHT [29] is $O\left(\alpha_{0} m^{2}\right)$, where the input image is with the size of $m \times m$, and $\alpha_{0}$ is the constant specifying per pixel complexity of DWT for a wavelet filter of a specific length. Therefore, the total complexity of the proposed combined model is $O\left(2 n \log (n / \varepsilon)+\alpha_{0} m^{2}\right)$. For the exhaustive search, the total complexity including SPIHT is $O\left((n !)\left(\alpha_{0} m^{2}\right)\right)$.

\section{Conclusion and Future Works}

This paper presented the distortion analysis and the bit allocation framework for multiview image coding with disparity-compensated wavelet lifting technique. The distortion prediction of multiview image coding is analyzed. The new rate distortion model combining the exponential and power models was proposed. Using the derived distortion and the proposed rate-distortion model, the bit allocation method among subbands was described. Experimental results showed that the proposed rate-distortion model can approximate the actual rate-distortion characteristics of image very well in a wide range of target bit rate. Moreover, using the proposed rate-distortion model in the rate allocation problem for coding multiview images provides close results of the optimal bit allocation and PSNR to the multiview image coding using the exhaustive search for the optimal bit rate allocation.

There are several possible future research extensions. First, the proposed framework can be further extended to multiview video both in distortion analysis and bit rate allocation. The framework of distortion analysis and bit allocation for multiview video will be more complex than multiview image because we need to consider all video frames in both the same and different views. Second, more advance rate distortion models can be considered to improve the model-based bit allocation to obtain the coding results as close to the exhaustive search as possible. Third, 
distortion analysis and bit allocation of multiview image and video coding using multireference frames (i.e., more than two frames as used in 5/3 disparity wavelet lifting) are interesting issues because using multireference frames can improve the compression ratio of multiview image and video compression. Finally, the distortion analysis and bit allocation framework of multiview image and video can be further investigated in another transform domain such as DCT domain and in industrial image and video compression standards such as JPEG-2000 or H.264.

\section{Acknowledgments}

This paper is partly supported by Panasonic Singapore Laboratories (SPL) and Faculty of Engineering, King Mongkut's University of Technology, Thonburi via National Research University Research Fund of Thai Government.

\section{References}

[1] X. Tong and R. M. Gray, "Coding of multi-view images for immersive viewing," in Proceedings of the IEEE International Conference on Acoustics, Speech and Signal Processing (ICASSP '00), vol. 4, pp. 1879-1882, Istanbul, Turkey, June 2000.

[2] M. Magnor, P. Ramanathan, and B. Girod, "Multi-view coding for image-based rendering using 3-D scene geometry," IEEE Transactions on Circuits and Systems for Video Technology, vol. 13, no. 11, pp. 1092-1106, 2003.

[3] A. Secker and D. Taubman, "Motion-compensated highly scalable video compression using an adaptive 3D wavelet transform based on lifting," in Proceedings of the IEEE International Conference on Image Processing (ICIP '01), vol. 2, pp. 1029-1032, October 2001.

[4] L. Luo, J. Li, S. Li, Z. Zhuang, and Y. Q. Zhang, "Motion compensated lifting wavelet and its application in video coding," in Proceedings of the IEEE International Conference on Multimedia and Expo (ICME '01), vol. 2, pp. 365-368, August 2001.

[5] B. Pesquet-Popescu and V. Bottreau, "Three-dimensional lifting schemes for motion compensated video compression," in Proceedings of the IEEE International Conference on Acoustics, Speech and Signal Processing (ICASSP '01), vol. 3, pp. 17931796, May 2001.

[6] C.-L. Chang, X. Zhu, P. Ramanathan, and B. Girod, "Interview wavelet compression of light fields with disparitycompensated lifting," in Visual Communications and Image Processing, vol. 5150 of Proceedings of SPIE, pp. 694-706, Lugano, Switzerland, July 2003.

[7] B. Girod, C.-L. Chang, P. Ramanathan, and X. Zhu, "Light field compression using disparity-compensated lifting," in Proceedings of the IEEE International Conference on Acoustics, Speech and Signal Processing (ICASSP '03), vol. 4, pp. 760-763, April 2003.

[8] A. Said and W. A. Pearlman, "A new, fast, and efficient image codec based on set partitioning in hierarchical trees," IEEE Transactions on Circuits and Systems for Video Technology, vol. 6, no. 3, pp. 243-250, 1996.

[9] N. Anantrasirichai, C. N. Canagarajah, and D. R. Bull, "Multiview image coding with wavelet lifting and in-band disparity compensation," in Proceedings of the IEEE International Conference on Image Processing (ICIP '05), vol. 3, pp. 33-36, September 2005.
[10] P. Lasang, W. Kumwilaisak, and A. Kaewpunya, "Multiview image coding via image feature matching and adaptive disparity-compensated wavelet lifting technique," in Proceedings of the 25th Picture Coding Symposium (PCS '06), Beijing, China, April 2006.

[11] Y. Shoham and A. Gersho, "Efficient bit allocation for an arbitrary set of quantizers," IEEE Transactions on Acoustics, Speech, and Signal Processing, vol. 36, no. 9, pp. 1445-1453, 1988.

[12] L.-J. Lin and A. Ortega, "Bit rate control using piecewise approximated rate-distortion characteristics," IEEE Transactions on Circuits and Systems for Video Technology, vol. 8, no. 4, pp. 446-459, 1998.

[13] J. Li, P.-Y. Cheng, and C.-C. J. Kuo, "Embedded wavelet packet image coder with fast rate-distortion optimized decomposition," in Visual Communications and Image Processing, vol. 3024 of Proceedings of SPIE, pp. 1077-1088, San Jose, Calif, USA, February 1997.

[14] N. M. Rajpoot, "Model based optimal bit allocation," in Proceedings of the Data Compression Conference (DCC '04), p. 558, March 2004.

[15] T. G. Cover and J. A. Thomas, Elements of Information Theory, John Wiley \& Sons, New York, NY, USA, 1991.

[16] S. Mallat and F. Falzon, "Analysis of low bit rate image transform coding," IEEE Transactions on Signal Processing, vol. 46, no. 4, pp. 1027-1042, 1998.

[17] W. Sweldens, "The lifting scheme: a construction of second generation wavelets," SIAM Journal on Mathematical Analysis, vol. 29, no. 2, pp. 511-546, 1998.

[18] N. Mehrseresht and D. Taubman, "Adaptively weighted update steps in motion compensated lifting based scalable video compression," in Proceedings of the IEEE International Conference on Image Processing (ICIP '03), vol. 3, pp. 771-774, September 2003.

[19] B. Girod and S. Han, "Optimum update for motioncompensated lifting," IEEE Signal Processing Letters, vol. 12, no. 2, pp. 150-153, 2005.

[20] H.-M. Hang and J.-J. Chen, "Source model for transform video coder and its application-part I: fundamental theory," IEEE Transactions on Circuits and Systems for Video Technology, vol. 7, no. 2, pp. 287-298, 1997.

[21] T. Rusert, K. Hanke, and J.-R. Ohm, "Transition filtering and optimized quantization in interframe wavelet video coding," in Visual Communications and Image Processing, vol. 5150 of Proceedings of SPIE, pp. 682-693, Lugano, Switzerland, July 2003.

[22] R. H. Byrd, M. E. Hribar, and J. Nocedal, "An interior point algorithm for large-scale nonlinear programming," SIAM Journal on Optimization, vol. 9, no. 4, pp. 877-900, 1999.

[23] R. H. Byrd, J. C. Gilbert, and J. Nocedal, "A trust region method based on interior point techniques for nonlinear programming," Mathematical Programming A, vol. 89, pp. 149-185, 2000.

[24] R. H. Byrd, J. C. Gilbert, and R. A. Tapia, "A trust region method based on interior point techniques for nonlinear programming," Report OTC 96/02, Optimization Technology Center, Northwestern University, Evanston, Ill, USA, 1996.

[25] http://cat.middlebury.edu/stereo/data.html.

[26] ftp://ftp.ne.jp/KDDI/multiview.

[27] F. Bellifemine, A. Capellino, A. Chimienti, R. Picco, and R. Ponti, "Statistical analysis of the 2D-DCT coefficients of the differential signal for images," Signal Processing: Image Communication, vol. 4, no. 6, pp. 477-488, 1992. 
[28] G. Bjontegaard, "Calculation of average PSNR differences between RD-curves," ITU SG16 VCEG-M33, April 2001.

[29] N. Splrjan, S. Grgic, and M. Grgic, "Modified SPIHT algorithm for wavelet package image coding," Real-Time Imaging, vol. 11, pp. 378-388, 2005. 